\title{
Novel Signalling Based Approach for Handling Linear and Non-Linear Impairments in Transparent Optical Networks
}

\author{
Invited paper
}

\author{
V. S. Chava, E. Salvadori, A. Zanardi, \\ Create-Net \\ Via Alla Cascata 56D, Trento, Italy 38100 \\ saradhi@ieee.org
}

\author{
G. Galimberti, G. Martinelli, R. Pastorelli, E. S. \\ Vercelli, A. Tanzi, D. La Fauci \\ Cisco Photonics, Via Philips 12, Monza, Italy 20052 \\ ggalimbe@cisco.com
}

\begin{abstract}
In GMPLS-based transparent optical networks, the switching and routing functionalities are performed in optical domain. However in these networks, physical layer impairments (PLIs) incurred by non-ideal transmission media accumulate along an optical path, and the overall effect determines the feasibility of lightpaths. Introducing transparency in optical layer reduces the possibility of GMPLS protocols interaction with optical layer at intermediate nodes along the path. Hence there is an urgent need to develop techniques that provide PLI information to GMPLS protocols and algorithms that efficiently utilize this information to realize dynamically reconfigurable GMPLS/WDM networks. We propose an optical control plane (OCP) architecture based on extensions to RSVP-TE, which is capable of handling both linear impairments (LIs) and non-linear impairments (NLIs). We propose a method to deal with the possible disruption of existing lightpaths because of excessive crosstalk introduced due to new lightpath setup. The simulation results suggest that $\mathrm{OCP}$ architectures to handle NLIs are important to avoid potential lightpath disruption.
\end{abstract}

Keywords- optical impairments, quality of transmission, impairment-aware routing, GMPLS control plane, RSVP-TE

\section{INTRODUCTION}

In transparent WDM optical networks a connection is referred to as a lightpath, and is established between any two nodes by allocating the same wavelength on all links along the chosen route. In these networks, if a lightpath is established between any two nodes, traffic between these nodes can be routed without requiring any intermediate optical-electricaloptical (OEO) conversion and buffering. However, physical layer impairments (PLIs) incurred by non-ideal optical transmission media accumulate along an optical path, and determine the feasibility or quality of transmission (QoT) of the lightpaths [1]. If the received signal quality is not within the receiver sensitivity threshold, the receiver may not be able to correctly detect the optical signal, causing the lightpath (and the corresponding reserved resources) to be useless.

A recent approach to network control and management using the GMPLS framework [2] developed by Internet engineering task force (IETF) emerging as the winning control plane solution for the next-generation optical networks. One of the main applications of GMPLS in the context of optical networks is the dynamic establishment of lightpaths. However, it suffers from a lack of physical layer details (e.g. PLIs, transponder characteristics and availability, etc). PLI variations arise due to two reasons: (a) aging or environmental conditions (e.g., temperature, seasons, stress, bending, etc.) which cause slow variations and are not network state-dependent, and (b) linear and nonlinear effects that are network-state dependent. In dynamic lightpath establishment scenarios, where the average setup time is very small; PLI changes due to (a) can be considered negligible. However, network state-dependent impairments such as NLIs and linear crosstalk may be significant. Hence, whenever there is a change in network status (e.g. lightpath setup/teardown), it may need to be communicated to all nodes. The availability of this up-to-date information is essential for a GMPLS-capable node to evaluate the effects of PLIs and to decide whether a proposed lightpath is feasible in the optical domain. Hence, the focus of this paper is to develop novel optical control plane (OCP) architectures and algorithms, based on signalling protocol (RSVP-TE) extensions, which are capable of handling both LI and NLIs.

\section{RELATED WORK}

In the literature, two broad approaches are proposed for impairment-aware routing in GMPLS-based transparent optical networks: centralized and distributed [3, 4]. Centralized approaches assume availability of a centralized server that is reachable by all network elements (NEs) and aware of complete network topology, resource availability and physical layer parameters through traffic engineering database (TED), which are used during routing and wavelength assignment (RWA). Hence, centralized approaches are able to guarantee a set of lightpath requirements (e.g. bandwidth, diversity, QoT, latency, etc.). However, the centralized approaches have scalability, flexibility, and interoperability problems [3, 4]. Hence in this study we focus on distributed approaches. 
Distributed approach is performed via all NEs in the network using a common distributed GMPLS control plane which manages the required procedures for establishing lightpaths. There are two different mechanisms under the distributed approach: a) signalling-based optical control plane (S-OCP) $[5,6]$ extends the RSVP-TE protocol to include PLI information. Each node computes a route using standard OSPFTE protocol without the knowledge of PLIs and then RSVP-TE carries PLI information along the route till the destination node, which evaluates the optical feasibility and establishes the lightpath; b) Routing-based OCP (R-OCP) [4, 7] introduces PLI information into the routing protocol such as OSPF-TE. By flooding link state advertisements (LSAs) enhanced with PLI information, all nodes populate their TED, thereby allowing each node to compute a feasible route while standard RSVP-TE is used for lightpath establishment.

Recently, there are a few studies on S-OCP $[5,6]$ and ROCP $[4,7]$ approaches. R-OCP approaches need several modifications to standard OSPE-TE to disseminate wavelength availability and PLI information. In an ideal network scenario, $\mathrm{R}-\mathrm{OCP}$ is expected to perform better compared to S-OCP. However, in real-world scenario, where there is some delay in updating PLI information R-OCP suffers from TED inconsistency, scalability, and stability [6]. R-OCP requires a complex CPU at each NE and takes longer time to solve multiconstraint routing problem [6]. S-OCP approach does not require global flooding of wavelength availability and PLI information; hence it efficiently handles frequent network changes and minimizes scalability problems. Since PLIs are updated at each node along the path and the optical feasibility is evaluated at the destination node, it is not necessary to have unified mathematical models at all the nodes. Furthermore, since no complex route computation algorithms are used, the load on NE's CPU is less. However, S-OCP approach suffers from larger path setup time due to increased number of setup attempts and sub-optimal resource allocation due to the usage of PLI-unaware route computation algorithms. Because of several advantages of S-OCP approach, in this study we use $K$ sequential S-OCP [6] to handle both LIs and NLIs.

\section{PHYSICAL LAYER IMPAIRMENT MODELING}

In general PLIs are classified as linear and non-linear impairments (LI and NLIs). However, PLIs can be split also into two main classes: single-channel effects and multichannels effects. The single-channel effects can be fully determined considering only the channel characteristics (e.g. bitrate, wavelength, modulation format, etc.) and the path characteristics (e.g. fiber loss, chromatic dispersion, amplifiers power, etc.). Both characteristics are in general static and therefore the single-channel effects can be calculated only once. On the contrary, the multi-channels effects depend not only on the channel and path parameters, but also on other lightpaths deployed in the network at the specific time and sharing some network sections.

\section{A. Modeling of Linear and Non-Linear Impairments}

LIs considered in our study include: loss, amplifier spontaneous emission (ASE) noise, polarization mode dispersion (PMD), chromatic dispersion (CD), polarization dependent loss (PDL), and crosstalk. In this study we use same LIs modeling as described in [6]. NLIs considered in our study include: self-phase modulation (SPM), cross-phase modulation (XPM), and four wave mixing (FWM). NLIs such as stimulated Brillouin scattering (SBS) and stimulated Raman scattering (SRS) are assumed to be taken care of during the network design phase. As detailed modeling of NLIs is very complex and space consuming we provide a brief overview of their modeling:

SPM: is computed by evaluating optical signal evolution along the path using split-step numerical method (with pattern bit length and samples per bit depending on transponder interface). At the destination node, the optical signal eye opening is converted to OSNR penalty using empirical formulae.

XPM: Total crosstalk (XT) due to XPM on a lightpath is approximated as the linear sum of XT contribution in each path section due to each adjacent active channel (i.e., $\sum_{i} \sum_{j}$ $X P M_{X T j}$, where $j$ is active channels in a path section and $i$ is path sections along the route) within the specific wavelength range depending on the fiber type. The XT contribution due to XPM for each path section is computed using empirical formulae.

FWM: Total XT due to FWM on a lightpath is computed as the linear sum of XT contribution in each path section along the route considering all adjacent active channels within the specific wavelength range depending on the fiber type. The XT contribution due to FWM for each path section is computed using empirical formulae.

\section{B. Overall Impairment and Optical Feasibility Evaluation}

When the PATH message arrives at the destination node each wavelength in the label-set is evaluated for optical feasibility. First, the wavelengths are evaluated considering only LIs. Let $O S N R_{A S E}$ be the OSNR at the receiver site, computed taking into account optical noise introduced along the path. The minimum required OSNR after considering LIs, $O S N R_{\text {lin }}$ is calculated using:

$$
\mathrm{OSNR}_{\text {lin }}=\mathrm{OSNR}_{\mathrm{txp}}+\mathrm{f}_{\mathrm{OSNR}}(\mathrm{PDL})+\mathrm{g}_{\mathrm{OSNR}}(\mathrm{PMD})
$$

where $O S N R_{t x p}$ is the target OSNR for the selected transponder without any impairments, $f_{O S N R}($.$) and g_{O S N R}($.$) are OSNR$ penalty due to PDL and PMD, respectively. If $O S N R_{A S E}$ is within the receiver thresholds, i.e., $O S N R_{A S E} \geq O S N R_{l i n}$, then the wavelength is considered as feasible w.r.t LIs. Then, for wavelengths that are feasible w.r.t LIs, the OSNR penalty due to optical devices (e.g. OXC switching matrix) and NLIs is computed and added to $O S N R_{\text {lin }}$ to obtain final minimum OSNR target $\left(O S N R_{\text {fin }}\right)$ as follows:

$$
\mathrm{OSNR}_{\text {fin }}=\mathrm{OSNR}_{\text {lin }}+\mathrm{OSNR}_{\mathrm{CD} / \mathrm{SPM}}+\mathrm{p}\left(\mathrm{XT}_{\mathrm{dev}}+\mathrm{XT}_{\mathrm{XPM}}+\mathrm{XT}_{\mathrm{FWM}}\right)
$$

where $O S N R_{\text {lin }}$ is minimum required OSNR after considering LIs; $O S N R_{C D S P M}$ is OSNR penalty due to linear CD and SPM; $X T_{\text {dev }}$ is XT contribution due to optical devices; $X T_{X P M}$ is the $\mathrm{XT}$ due to XPM; $X T_{F W M}$ is XT due to $\mathrm{FWM} ; p($.$) is empirical$ formulae, to evaluate OSNR penalty due to XT from optical devices, XPM, and FWM. All empirical formulae are obtained 
from theoretical studies and experimental validation and considered as Intellectual Property, hence not included here.

If the $O S N R_{A S E}$ is within the working area of the transponder, i.e., $O S N R_{A S E} \geq O S N R_{\text {fin }}$, the wavelength is considered as feasible w.r.t both LIs and NLIs; otherwise it is considered as not feasible. Note that by doing impairment evaluation in two steps as explained, we can immediately reject the lightpath requests that are not feasible w.r.t LIs without evaluating complex and time consuming OSNR penalties due to NLIs. It will help in reducing the setup time, as the source node can try on next candidate route avoiding NLIs evaluation. After evaluating whether the new lightpath is feasible, control plane protocols have to make sure that the establishment of new lightpath will not disrupt the existing lightpath by introducing excessive crosstalk due to non-linear or multichannel effects as discussed in Section. IV. Finally, if the new lightpath is feasible and no active lightpaths are disrupted then the lightpath is setup.

\section{CONTROL Plane PROTOCOLS AND TECHNIQUES FOR HANDLING LI AND NLIS}

In this section, we explain signalling based OCP architecture that can handle both LI and NLIs, called NLI-OCP, required protocols and corresponding extensions.

\section{A. Extended RSVP-TE Working Procedure}

For a new connection request, the source node computes $k$ explicit routes. The signalling process checks the optical feasibility of first explicit route by sending out a $P A T H$ message containing several fields describing LIs (e.g. OSNR, power, CD, PMD, XT, etc.,) and NLIs (e.g. path section characteristics such as signal power, fiber type, and length; signal type such as modulation format and bit-rate; adjacent active channel characteristics such as wavelength, LSP ID, crosstalk margin, residual CD, reference FWM XT) and a list of available transponders/wavelengths. Upon reception of $P A T H$ message, each intermediate node updates these fields considering wavelength continuity constraint and active adjacent channels on the path section. If there is no free wavelength on its outgoing link, then it sends PATH_ERR message to source node. If the destination node receives $\bar{P} A T H$ message, it evaluates OSNR penalty due to LIs. Then it evaluates the effect of active lightpaths on new lightpath only for the wavelengths that are feasible w.r.t LIs. Then the extra margin compared to the selected transponder interface called crosstalk margin $(X T m)$ is computed as follows:

$$
\begin{aligned}
& \mathrm{XTm}=\mathrm{p}^{-1}\left(\min \left\{\left[\mathrm{OSNR}_{\mathrm{ASE}}-\left(\mathrm{OSNR}_{\mathrm{lin}}+\mathrm{OSNR}_{\mathrm{SPM} / \mathrm{CD}}\right)\right], \mathrm{Max}_{-} \mathrm{XT}_{\mathrm{pen}}\right\}\right) \\
& -\left(\mathrm{XT}_{\mathrm{dev}}+\mathrm{XT}_{\mathrm{XPM}}+\mathrm{XT}_{\mathrm{FWM}}\right)
\end{aligned}
$$

$X T m$ indicates additional XT that can be introduced by future lightpaths without exceeding the transponder threshold or disrupting the lightpath. Max_XTpen is the maximum tolerated OSNR penalty for multi-channel effects and $p^{-1}($. inverse empirical formulae of $p($.).

Then the effect of new lightpath on active lightpaths is computed. It represents the additional XT that is introduced by the new lightpath on existing lightpaths, which is called additional crosstalk ( $a d d X T)$. It is a sum of XT contributions due to XPM and FWM computed per each path section that is common with the new lightpath, i.e., $a d d X T=\sum_{i} X P M_{X T}+\sum_{i}$ $F W M_{X T}$, where $i$ is all path sections that are common for both active and new lightpath. Whether addXT can be accommodated, i.e., (XTm-addXT $\geq 0)$, on all the active adjacent lightpaths is evaluated using the parameters collected during the PATH message. However, if some LSPs are setup or torn-down in the mean time (time between the collection of optical parameters during $P A T H$ message and the time $R E S V$ message reaches the same node) this check is not valid and may lead to 1) potential disruption of existing lightpaths and/or 2) potential rejection of new lightpath due to unavailability of $X T m$ on existing active channels. Hence, there is a need for a mechanism to handle the network status changes between $P A T H$ and RESV messages and is handled by $X T$ reservation protocol as described in Section IV. B.

After evaluation of both effects, i.e., effect of active lightpaths on new lightpath and effect of new lightpath on active lightpaths, if 1) $X T m \geq 0$ for new lightpath and 2) XTmaddXT $\geq 0$ for all active lightpaths that have some common path sections with new lightpath, then the destination node sends RESV message with a selected wavelength and transponder pair, otherwise, it sends back a PATH_ERR message. Upon reception of $R E S V$ message, intermediate nodes configure the OXC and stores LSP optical parameters such as $X T m$, section residual $\mathrm{CD}$, section reference $\mathrm{FWM}$, and signal/transponder type, and triggers XT reservation protocol (described in Section IV.B) to deduct addXT from active LSPs $X T m$. This additional check if $X T m$-addXT $\geq 0$ using XT reservation protocol is done at destination nodes of affected LSPs to handle network status changes. To perform this additional check $X T_{-} R E S V \_R E Q$ carries updated parameters required. If XT reservation for all affected active LSPs is successful, it forwards $R E S V$ to the next upstream node. In case if there are no active LSPs on certain path section there is no $\mathrm{XT}$ reservations triggered at the corresponding intermediate node. If XT reservation on some active lightpaths fails for some reason (e.g. the network status is changed) the $R E S V$ is rolled back using $R E S V$ ERR message. If source node receives the $R E S V$, then it will transmit data on the first route. If source node receives $P A T H E R R$ message, it will send $P A T H$ message on the second explicit route and repeat the process until all $k$ routes have been tried.

\section{B. XT Reservation Protocol Working Procedure}

The management of $X T m$ of any existing lightpath, say $L S P i$, and the reservation/deduction of addXT due to new lightpath from active lightpath $X T m$, say $L S P n$, are performed in a sequential manner by the destination nodes of each affected lightpath $L S P i$. Before allocating a channel or configuring $\mathrm{OXC}$ for $L S P n$ during $R E S V$ process in a path section on which there are some existing lightpaths that may potentially be disrupted by $L S P n$, the transit node requests (with message $X T \_R E S V \_R E Q$ ): a) reservation of $a d d X T$ on the affected $L S P i$ destination node; addXT is computed from the $L S P i$ parameters stored in the transit node during LSPi RESV processing, b) reservation of addXT to $L S P n$ destination node itself, if $L S P i$ channel was not considered in the original $P A T H$ message (may be because $L S P i$ has been setup during LSPn 
$P A T H$ message processing and therefore LSPi's effect on the $L S P n$ is not considered during its impairment evaluation); addXT is computed from the $L S P n$ parameters carried in $L S P n$ $R E S V$ message, $\mathrm{c}$ ) reservation for all affected lightpaths, i.e., all $L S P i$ 's, and also for $L S P n$ must be successful to allocate and configure $L S P n$; if one XT reservation fails, the setup is rolled back using $R E S V \_E R R$ message. Once the $X T_{-} R E S V \_R E Q$ reaches the $L S P i$ 's destination node it allows XT reservation if the sum of all active reservations and requested $a d d X T$ is less than available $X T m$ (i.e., $X T m \geq \sum_{x} a d d X T_{x}+a d d X T$ where $x$ is the previous addXT reservations). The effected destination node clears addXT if it is explicitly requested for example because of LSP tear-down.

\section{RSVP-TE Extensions to Handle NLIs}

To encompass LIs and NLIs, standard RSVP-TE signalling messages are extended to carry information required to evaluate optical feasibility. The following information is required in the $P A T H$ message: 1) requested service/traffic parameters: a) service type such as encoding type, payload type, etc., b) service QoS such as bandwidth, etc., c) extended service optical parameters such as transponder type, etc. 2) signalled path parameters: d) path parameters/QoS such as hop number, minimum latency, etc., e) extended path optical parameters (e.g., OSNR, power, CD, PMD, PMD, and XT). 3) path resources and status: f) label-set, g) path sections description such as fiber type, length, input power, input CD, etc., h) path sections active channels such as active LSP IDs, transponder type, $X T m$, residual $\mathrm{CD}$, reference FWM XT, etc.

The standard GENERALIZED_LABEL_REQUEST [8] and $T S P E C / F L O W \_S P E C$ [8-10] objects support encoding of (a) and (b). Standard one pass with advertising (OPWA) information is carried in the $A D S P E C$ object [9] encodes (d). Standard LABEL_SET object [8] with GENERALIZED_LABEL encodes (f). The encoding of (c), (e), (g), and (h) requires extensions to the standard $P A T H$ message and are encoded in LSP REQUIRED ATTRIBUTES object [11-13]. Note that $P A T \overline{T H}$ message size increases due to the addition of path section information and affected channel information at each hop. The $P A T H$ message size can easily exceed the maximum transfer unit (MTU) and may lead to message fragmentation. Large MTU values [14] or jumbo frames [15] may also be exceeded in worst case scenarios. A possible solution to message fragmentation is described in [12], which splits $P A T H$ message into multiple messages.

The information is required in RESV message is: selected channel information such as, channel identifier, and link physical properties able to evaluate all LIs and NLIs (e.g., CD, $X T m$, per section reference FWM XT), etc. We encoded this information in LSP_REQUIRED_ATTRIBUTES object [1113]. The standard RSVP-TE behavior has also been extended to trigger $X T \_R E S V \_R E Q$ to reserve $X T m$ on affected lightpaths when a new lightpath is setup.

To manage the $X T m$ on affected channels we have defined a new protocol with following three messages: 1) $X T \_R E S V \_R E Q$ is defined to reserve $X T m$ on affected channels and new channel (in case of changes in network status) destination nodes. It contains affected and affecting
LSP IDs, XPM XT, and new FWM XT. 2) XT RESV RESP is defined to get response from the affected channels destination node and it only contains whether XT_RESV_REQ is successful or not. 3) $X T R E S V R E L$ is defined to release $X T m$ on affected channels destination node and it contains same information as $X T_{-} R E S V_{-} R E Q s$.

\section{CONTROL Plane PRotocols AND TECHNIQUES FOR HANDLING LI AND NLIS}

In this section, we present simulation environment and network/traffic scenarios used in simulation experiments. Three different OCP architectures are compared w.r.t several performance metrics. The three architectures are: 1) LIs only $(L I-O C P)$ - in which only LIs considered in feasibility evaluation assuming that NLIs are considered during design phase [6]; 2) LIs with additional constraints (LI-ADD-OCP) in which LIs are considered together with worst case OSNR penalty to compensate for XT due to NLIs during feasibility evaluation; 3) LIs and NLIs (NLI-OCP) - in which both LI and NLIs are considered in feasibility evaluation.

The $K$-SEQ S-OCP architecture as described in Section IV together with RSVP-TE extensions to encompass PLIs, i.e., both LIs and NLIs, and XT reservation protocol has been implemented in GMPLS lightwave agile switching simulator (GLASS) [16]. In addition, a statistical processing module ANCLES [17] has been integrated to collect simulation results with target accuracy of 0.05 and confidence level of $95 \%$. When a node receives a connection request, the $k-C S P F$ algorithm computes $k$ routes. The CSPF metric used for route computation is number of hops with $K=3$. LIs and NLIs modeling and feasibility evaluation is done as described in Section III. Finally, feasible wavelength is selected using firstfit WA. In general, each node takes some time to process various RSVP-TE messages and to setup/clear OXC configuration. To simulate real-world scenario, we have considered the following: 1) PATH message processing time = 10 msec; 2) Egress node (where time consuming LI and NLIs evaluation is carried out) $P A T H$ message processing time $=$ $100 \mathrm{msec}$; 3) RESV, PATH ERR, RESV ERR message processing time is $=10 \mathrm{msec} ; \overline{4}) X T \_R E S V_{-} \bar{R} E Q$ processing time $=10 \mathrm{msec}$; and 5) $\mathrm{OXC}$ switch configuration time $=50$ msec. In $L I-A D D-O C P$ simulation scenario, the network is over dimensioned with extra OSNR penalty considering worst case XT due NLIs. Additional constraints introduced are: 1) additional OSNR penalty: $1.5 \mathrm{~dB}, 2$ ) maximum number of hops: a) 7 hops on edge links; or b) max 4 hops edge links plus 1 hop on inner links; or c) max 2 hops on edge links plus 2 hops on inner links; or d) max 3 hops on inner links.

\section{A. Network and Traffic Scenarios}

Several simulation experiments have been performed on regular networks such as Grid $4 \times 4$ and Daisy chain as shown in Fig. 1. Due to space constraints we present the results for Grid $4 \times 4$. The simulation results for Daisy chain are similar. In Grid $4 \times 4$ topology 1) the border links are $50 \mathrm{Km}$ TrueWave Classic fibers (TWCF) with booster amplifier (BST) with output power of $-1 \mathrm{dBm}$ and pre-amplifier (PRE) $+1 \mathrm{dBm}$ 
and 2) the inner links are $60 \mathrm{Km}$ TWCF with BST with output power of $+1 \mathrm{dBm}$ and PRE at $+1 \mathrm{dBm}$. All networks are transparent and no wavelength converters have been considered. Each link has 2 unidirectional standard fibers and each fiber carries 40 wavelengths on Band $C$ with $100 \mathrm{GHz}$ spacing. As fiber links are not longer than $80 \mathrm{Km}$, only PRE and BST amplifiers are used at each node, while no in-line amplifiers are used on fiber links. Erbium-doped fiber amplifiers (EDFAs) are considered in simulations. A unique transponder is used with its characteristics calibrated to allow the setup of the longest shortest path in the network. Various optical parameters used in simulation experiments are given in Table 1.

TABLE 1: OPTICAL PARAMETERS USED IN SIMULATIONS

\begin{tabular}{|c|c|c|}
\hline Description & Value & Unit \\
\hline Output Power of Transmitter & 0 & $\mathrm{dBm}$ \\
\hline Loss of Fiber & 0.25 & $\mathrm{~dB} / \mathrm{km}$ \\
\hline Channel Add Loss & 8 & $\mathrm{~dB}$ \\
\hline Channel Drop Loss & 14.3 & $\mathrm{~dB}$ \\
\hline Channel Pass-through Loss & 12.9 & $\mathrm{~dB}$ \\
\hline Noise Figure of EDFA & 5.8 & \\
\hline Reference Wavelength & 1545.32 & $\mathrm{~nm}$ \\
\hline CD of TWCF at Reference Wavelength & 2.03 & $\mathrm{ps} / \mathrm{hm} / \mathrm{km}$ \\
\hline Slope Factor of CD of TWCF & 0.07 & $\mathrm{ps} / \mathrm{nm} / \mathrm{km}$ \\
\hline PMD of TWCF & 0.1 & $\mathrm{ps} / \mathrm{km}$ \\
\hline PMD of Optical Amplifier & 0.3 & $\mathrm{ps}$ \\
\hline PMD of Optical Nodes & 0.25 & $\mathrm{ps}$ \\
\hline Crosstalk Ratio of Optical Nodes & -35 & $\mathrm{~dB}$
\end{tabular}

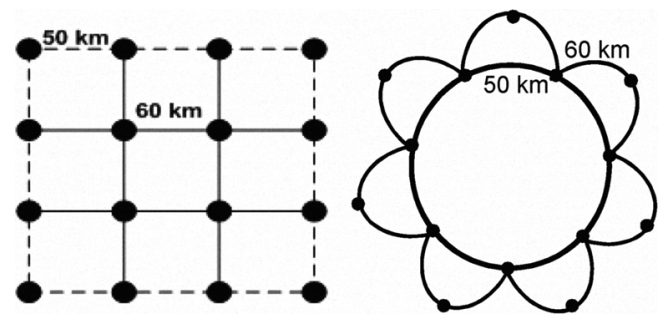

Figure 1: Topologies used in simulations a) Grid $4 \times 4$ and b) Daisy chain.

The traffic scenario consists of a classical traffic model based on lightpath requests with Poisson arrivals at an average rate $1 / \mu$ (with $\mu=2 \mathrm{sec}$ ) per second. An installed lightpath has an average exponential duration of $v \mathrm{sec}$. The traffic requests are uniformly distributed among all nodes. The traffic load $\beta$ is defined as the average network resource (wavelength) usage computed in percent as: $\beta=\left(N_{c} \times L_{c}\right) /(M \times W) \times 100 \%$, where $N_{c}$ is the average number of active connections and equals $v / \mu, L_{c}$ is the average number of hops in the network considering only shortest paths between all pairs of nodes, $M$ is the number of links and $W$ is the number of wavelengths in the network.

\section{B. Performance Metrics}

In this work, we have used following performance metrics:

A) Blocking probability (BP): The ratio of the number of rejected connection requests to all requested connections. Three main components that contribute to BP are: 1) blocking due to unavailability of free wavelength, called wavelength blocking, 2) blocking due to impairments, called optical blocking, 3) blocking due to reservation conflicts or XT reservation errors, called reservation blocking.

B) Lightpath setup time: Elapsed time between the first
$P A T H$ message sent and the RESV message received at the source node. This metric reflects how fast a connection request can be established.

\section{Comparison of LI-OCP, LI-ADD-OCP, and NLI-OCP}

The simulation results in this section are for average interarrival time, $I=2 \mathrm{sec}$. Fig. 2 shows BP of three architectures. From the results it is clear that adding additional constraints to encompass NLIs using $L I-O C P$ architecture leads to worse network performance especially at low network loads. At low network loads, additional constraints are too conservative and block more requests, while NLI evaluation allows connection setup. This confirms that introducing a higher complexity in OCP to encompass LIs and NLIs is worth, since it leads to better performance and to more relaxed network dimensioning criteria. As load increases, BP for NLI-OCP increases due to increase in effect of non-linearities. The effect of nonlinearities will finally approach the worst case additional constraint introduced, and hence BP for both $L I-O C P$ and NLI$O C P$ is same at very higher loads.

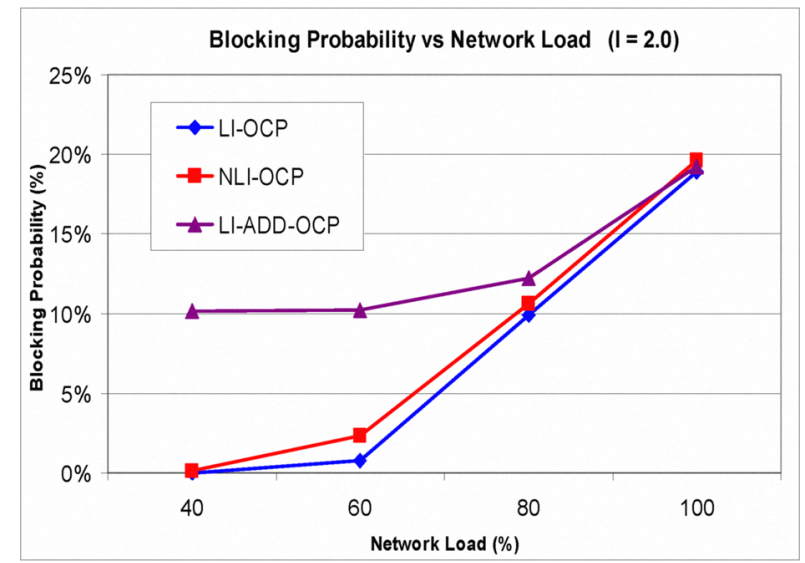

Figure 2: BP vs. load.

BP for $L I-O C P$ is less than that of NLI-OCP and $L I-A D D$ $O C P$ for all loads. Though it looks like good w.r.t network performance, it is not really true because of the following reasons. The overall BP of NLI-OCP mainly consists of blocking due to 1) LIs, 2) the effect of active lightpaths on new lightpath 3) the effect of new lightpath on existing lightpaths. As effects (2) and (3) are not considered in LI$O C P$, even if it is able accept more connections these lightpaths may not be optically feasible and hence the corresponding reserved resources may become useless. As $L I$ $O C P$ does not consider the effect (3), it may potentially disrupt existing lightpaths by introducing excessive XT. Hence $L I-O C P$ is not considered as good architecture.

For longer hop LSPs the BP is high as anticipated and it increases as load increases as shown in Fig. 3. At low network loads, the additional constraints block a great part of connection requests while NLI evaluation allows the connection setup. At high network loads, NLI-OCP will lead to higher BP because of different resource allocation in the network: as the longer LSPs are allowed, more wavelengths are allocated in the network and there is a higher failure rate 
due to wavelength continuity constraint. Again here the same arguments apply to $L I-O C P$ architecture as discussed earlier.

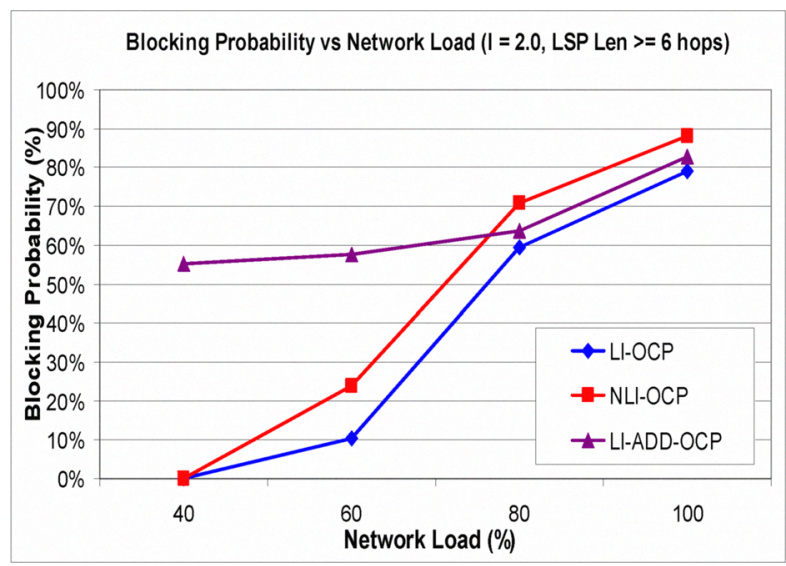

Figure 3: BP vs. load for LSPs with hop length $\geq 6$.

Fig. 4 shows the contribution of wavelength blocking, optical blocking, and reservation blocking to overall BP of $N L-O C P$. The highest contribution to overall $\mathrm{BP}$ is due to wavelength blocking and it increases with the load. Out of LSPs that are able to find a free common wavelength on all links along the route, the blocking is mainly due to NLIs, as network is designed such a way that there is no impact of LIs. The NLI blocking is in the range of $20-55 \%$ (inset of Fig. 8), at normal network operating loads of $50-75 \%$. Which is considered as very high thus motivates the need for handling NLIs with GMPLS enhancements. The reservation blocking is negligible for all loads.

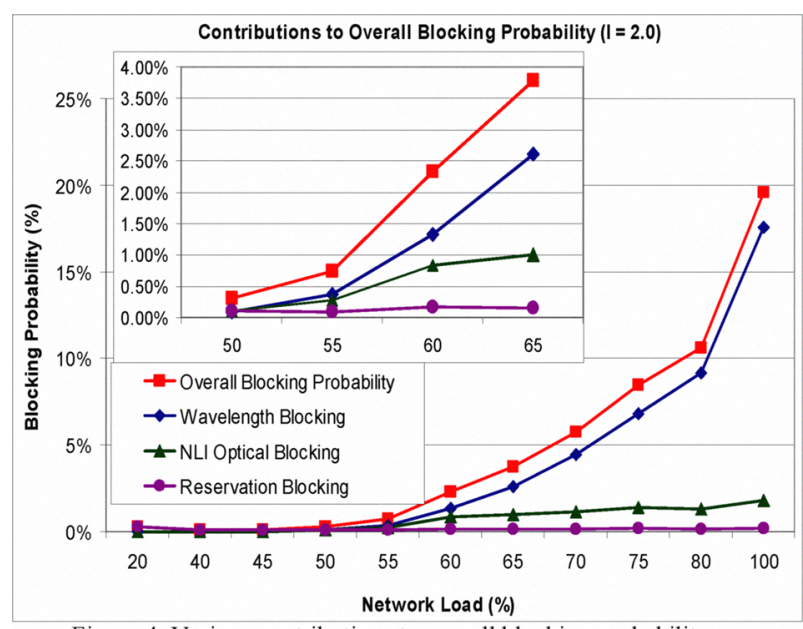

Figure 4: Various contributions to overall blocking probability

The blocking due to NLIs is further divided into 1) blocking due to effect of existing lightpaths on new lightpath and 2) blocking due to effect of new lightpath on existing lightpaths. To understand which of these effects dominate we conducted simulations and results are shown in Fig. 5. Though, individual effects do not follow any trend due to inherent nonlinearities which depend on the network status, resource allocation, and network load; it is clear that there is substantial blocking due to various components of NLI blocking. At normal network operating loads (50-75\%) the blocking due to effect of new lightpath on existing lightpaths is around 1.9$3.1 \%$. In other words, establishment of new lightpath disrupts $1.9-3.1 \%$ of existing lightpaths, if there is no mechanism to handle the effect of new lightpath on existing lightpaths. Note that effect of disrupting an active LSP is worse than not admitting a new LSP. This further motivates the need for GMPLS control plane enhancements for handling NLIs.

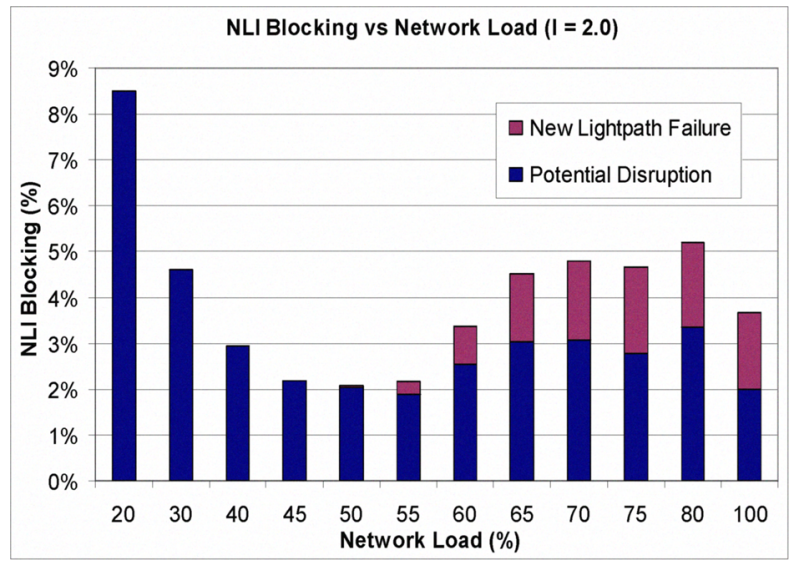

Figure 5: Percentage of failures due to various effects of NLIs.

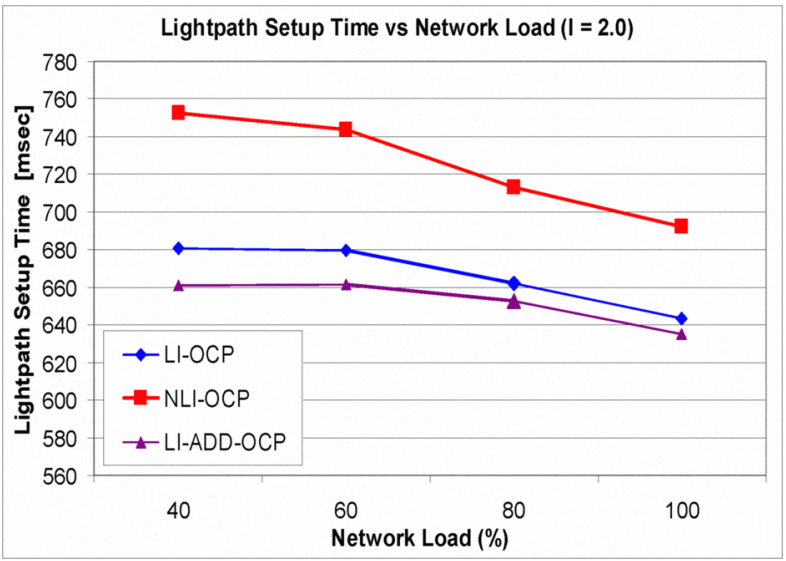

Figure 6: Average LSP setup time vs. load.

Average LSP setup time is shown in Fig. 6. The LSP setup time is mainly function of LSP length and number of attempts. In case of $L I-O C P$ the number of attempts is lower leading to lower setup time. While in case of NLI-OCP the set time is highest. As the main contribution is due to time it takes to evaluate NLIs at the destination node though the average number of hops and average number of attempts are close to that of $L I-O C P$. The average number of attempts in case of $L I$ $A D D-O C P$ is highest as it is over provisioned to consider worst case OSNR penalty; however, the average number of hops is the lowest because it can not reach long distances after considering worst case OSNR penalty. Hence the setup time for $L I-A D D-O C P$ is lowest of three architectures. In all cases, as load increases, 1) the average number of attempts increases 2) the average number of hops decreases. So the overall setup time decreases as the load increases. 


\section{CONCLUSIONS}

We developed novel GMPLS-based OCP architectures and algorithms that are capable of handling both LI and NLIs. We conducted extensive simulation experiments, to compare approaches that consider: only LIs, LIs with additional constraints to deal with NLIs, and both LIs and NLIs. Based on the simulation results and other considerations such as engineering complexity, modifications required to RSVP-TE, and feasibility of deployment, we have drawn the following conclusions: 1) NLI-OCP outperforms LI-OCP considering overall network performance and potential active lightpath disruption. 2) $L I-A D D-O C P$ leads to more blocking compared to $N L I-O C P$. 3) Since $L I-O C P$ needs network to be overdimensioned to get similar performance as $N L I-O C P$, it is worth implementing relatively complex $N L I-O C P$ to reap its benefits.

\section{ACKNOWLEDGMENT}

The authors would like to thank Sergio Dalsass for his help in collecting simulation results.

\section{REFERENCES}

[1] G. P. Agrawal, Fiber Optic Communication Systems. Wiley Interscience, 1992.

[2] A. Farrel and I. Bryskin, GMPLS: Architecture and Applications, Morgan Kauffman, 2005.

[3] P. Castoldi et al., "Centralized vs Distributed Approaches for Encompassing Physical Impairments in Transparent Optical Networks," Proc. of ONDM, pp. 68-77, May 2007.
[4] R. Martínez et al., "Challenges and Requirements for Introducing Impairment-Awareness into the Management and Control Planes of ASON/GMPLS WDM Networks," IEEE Communications Magazine vol. 44 , no. 12 , pp. $76-85$, Dec 2006.

[5] F. Cugini et al., "A Novel Signaling Approach to Encompass Physical Impairments in GMPLS Networks," Proc. of IEEE Globecom, 2004.

[6] E. Salvadori et al., "Distributed Optical Control Plane Architectures for Handling Transmission Impairments in Transparent Optical Networks," IEEE/OSA Journal of Lightwave Technology, vol. 27, no. 13, Jul 2009.

[7] J. Strand and A. Chiu, "Impairments and Other Constraints on Optical Layer Routing," RFC 4054, May 2005

[8] L. Berger, "Generalized Multi-Protocol Label Switching (GMPLS) Signaling Resource reSerVation Protocol-Traffic Engineering (RSVPTE) Extensions," RFC 3473, Jan 2003

[9] J. Wroclawski, "The Use of RSVP with IETF Integrated Services," RFC2210, Sep 1997.

[10] D. Papadimitriou, "Generalized Multi-Protocol Label Switching (GMPLS) Signaling Extensions for G.709 Optical Transport Networks Control", RFC4328, Jan 2006.

[11] A. Farrel et al., "Encoding of Attributes for Multiprotocol Label Switching Label Switched Path Establishment Using Resource reSerVation Protocol-Traffic Engineering," RFC 4420, Feb 2006.

[12] G. Martinelli, et al., "GMPLS Signaling Extensions for Optical Impairment Aware Lightpath Setup," IETF draft, Feb 2008.

[13] A. Farrel et al., "Encoding of Attributes for Multiprotocol Label Switching Label Switched Path Establishment Using Resource reSerVation Protocol-Traffic Engineering," IETF draft, May 2008

[14] R. Atkinson et al., "Default IP MTU for use over ATM AAL5," RFC 1626, May 1994

[15] J. Kaplan et al., "Extended Ethernet Frame Size Support," IETF draft, Nov 1999.

[16] GLASS simulator, http://www-x. antd nist.gov/glass/Main.htm.

[17] ANCLES simulator: http://www.telematica.polito.it/ancles 